\title{
A molecular dynamics study of the thermal transport in silicon/germanium nanostructures: From cross-plane to in-plane
}

\author{
Zuyuan Wang \\ Max Planck Institute for Polymer Research, Ackermannweg 10, 55128 Mainz, Germany
}

\section{A R T I C L E I N F O}

\section{Keywords:}

Thermal transport

Molecular dynamics

Interface

Semiconductor

\begin{abstract}
A B S T R A C T
Thermal transport in silicon/germanium $(\mathrm{Si} / \mathrm{Ge})$ nanostructures has been widely studied in both the cross-plane and in-plane configurations. It remains unknown, however, how heat transports in the intermediate configurations. Here we conduct nonequilibrium molecular dynamics simulations to study the thermal conductance of $\mathrm{Si} / \mathrm{Ge}$ nanostructures that gradually change from a cross-plane to an in-plane configuration. The thermal conductances of the cross-plane and in-plane $\mathrm{Si} / \mathrm{Ge}$ nanostructures are found to be $(1.57 \pm 0.01) \times 10^{8}$ and $(1.60 \pm 0.02) \times 10^{8} \mathrm{~W} / \mathrm{m}^{2}-\mathrm{K}$ respectively at $300 \mathrm{~K}$, both smaller than those of the intermediate nanostructures at the same temperature. As temperature increases, the thermal conductances of the nanostructures decrease, with those of the intermediate nanostructures exhibiting a stronger temperature dependence. The thermal conductances are inconsistent with the trend of the overlap of the vibrational density of states (VDOS) but agree well with the trend of the integrated VDOS in the middle region of the nanostructures.
\end{abstract}

Silicon/germanium $(\mathrm{Si} / \mathrm{Ge})$ nanostructures are important for many applications (e.g., electronics [1,2], photonics [3-5], sensors [6,7]), where appropriate thermal transport properties are critical for achieving the desired performance. Many studies have been carried out to understand the heat transfer in $\mathrm{Si} / \mathrm{Ge}$ nanostructures [8-18], especially those with the $\mathrm{Si}$ and Ge components arranged in either a crossplane $[8,11,14,16]$ or an in-plane $[17,18]$ configuration. Of particular interest are the studies on the thermal transport across interfaces, which have elucidated the effects of the roughness, interdiffusion, disorder, dislocations, and bonding on the thermal boundary conductance $[13,19,20]$. It has been shown that an enhanced phonon transmission can be explained by an increased overlap of the vibrational density of states (oVDOS) on both sides of the interface [13]. Yet, Zhang et al. have reported that the oVDOS is not the only reason affecting the thermal transport property, as a linearly increasing oVDOS with temperature does not corroborate a linearly increasing thermal conductivity [21]. Furthermore, it has been shown that temperature has no influence on the phonon transmission in $\mathrm{Si} / \mathrm{Ge}$ superlattices [14], which contradicts the well-known temperature-dependence of the thermal conductivity. Despite the many previous studies, it remains unknown how heat propagates in the $\mathrm{Si} / \mathrm{Ge}$ nanostructures with configurations intermediate between the cross-plane and in-plane ones. This information, however, is important not only for further elucidating the effects of the oVDOS and temperature on the thermal transport properties of the nanostructures but also for expanding the thermal design space for the aforementioned applications.

In this work, we use nonequilibrium molecular dynamics (NEMD) simulations to study the thermal conductances of $\mathrm{Si} / \mathrm{Ge}$ nanostructures, which have configurations varying gradually from cross-plane to inplane, over the temperature range from 300 to $1000 \mathrm{~K}$. We find that the intermediate configurations show up to $18 \%$ higher thermal conductances than the cross-plane and in-plane ones at room temperature and that the thermal conductances of all the configurations decrease with increasing temperature. Moreover, the thermal conductance results correlate well with the integrated vibrational density of states (intVDOS) in the middle region of the nanostructures.

Fig. 1(C1)-(C9) shows the nine thermal transport configurations considered in this work. Fig. 1(C1) illustrates an xyz coordinate system, the thermal reservoirs, and the Si and Ge material components. From $\mathrm{C} 1$ to $\mathrm{C} 9$, the amounts of the $\mathrm{Si}$ and Ge materials are kept the same, so is the number of interfaces, but the orientation of the interface gradually changes from vertical to horizontal. It should be noted that the interfaces considered in this work are atomically flat.

The simulations are conducted by using LAMMPS [22]. The initial structures of the simulated systems are generated by arranging the $\mathrm{Si}$ and $\mathrm{Ge}$ atoms in an f.c.c. lattice (in a diamond-like structure) with a uniform lattice constant $5.550 \AA$. Each structure contains 74, 16, and 16 unit cells (u.c.) in the $x, y$, and $z$ directions, respectively. The crystallographic directions in the three directions are all equivalent to [001]. Along the $x$ direction, the simulation domain is divided into five

E-mail address: wang.zuyuan@mpip-mainz.mpg.de. 


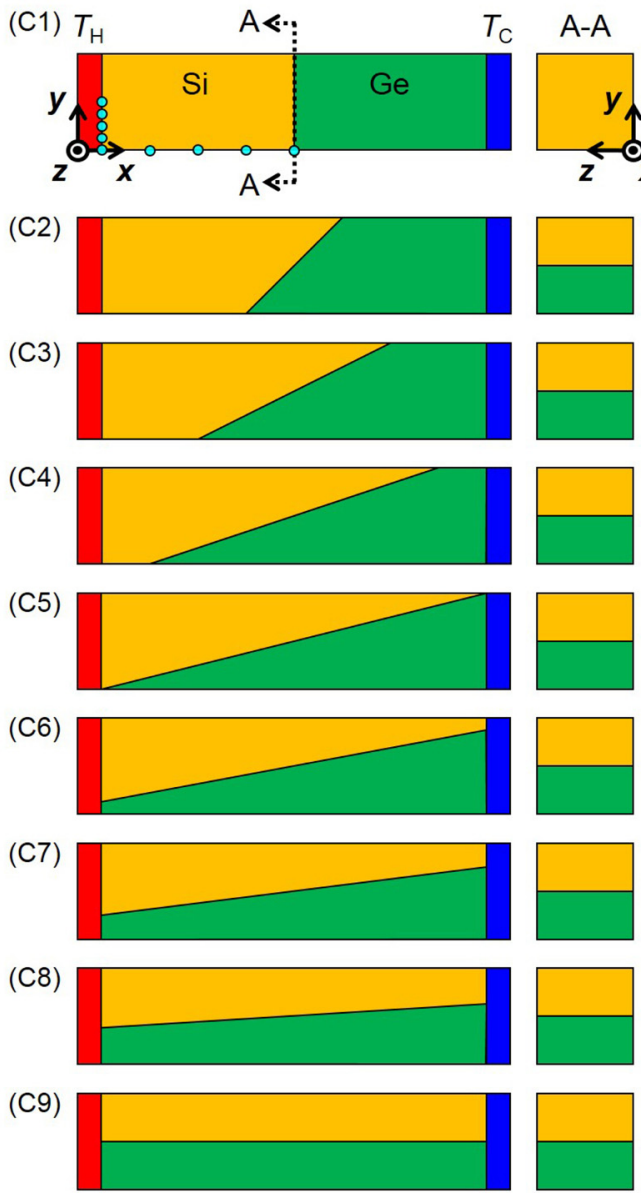

Fig. 1. Schematics of the simulation domains. (C1)-(C9) illustrate the nine thermal transport configurations, which gradually change from cross-plane to in-plane. In each configuration, both a front view and an "A-A" cross-sectional view are shown. The red and orange regions comprise silicon (Si) atoms; the green and blue regions comprise germanium (Ge) atoms. The red and blue regions represent hot $\left(T_{\mathrm{H}}\right)$ and cold $\left(T_{\mathrm{C}}\right)$ reservoirs, respectively. An $x y z$ coordinate system is shown in (C1). (For interpretation of the references to color in this figure legend, the reader is referred to the web version of this article.)

regions: a layer of fixed atoms on the left ( 1 u.c.), a hot reservoir (4 u.c.), a device region (64 u.c.), a cold reservoir (4 u.c.), and a layer of fixed atoms on the right (1 u.c.), as shown in Fig. 2(a). We apply free boundary conditions in the $x$ and $y$ directions and periodic boundary conditions in the $z$ direction. The temperatures of the hot and cold reservoirs are maintained at $T+30 \mathrm{~K}$ and $T-30 \mathrm{~K}$, respectively, with $T$ being the nominal temperature of the simulation [23]. The Si-Si, Ge-Ge, and Si-Ge interatomic interactions are characterized by the Tersoff potential [24]. The time step is $1 \mathrm{fs}$ for all simulations, which is sufficient to capture the highest vibrational frequencies in the considered nanostructures ( $\sim 16 \mathrm{THz})$ [25]. Each simulation is conducted by first equilibrating the material system in NPT for $0.2 \mathrm{~ns}$. Then the atoms at the two ends are fixed, and Berendsen thermostats [26] are used to maintain the temperatures of the hot and cold reservoirs at the desired values. After that, the simulation is conducted in NVE for 9 ns for data production. We note that upon equilibrium at $300 \mathrm{~K}$ the lattice constants of $\mathrm{Si}$ and $\mathrm{Ge}$ are 5.504 and $5.575 \AA$, respectively, in good agreement with the experimental values [27]. For each condition, three independent simulations are performed and the average results and their standard deviations are reported. It has been shown that NEMD simulations have much smaller uncertainties than EMD simulations [28].

Fig. 2(b) shows typical temperature profiles from the simulations.
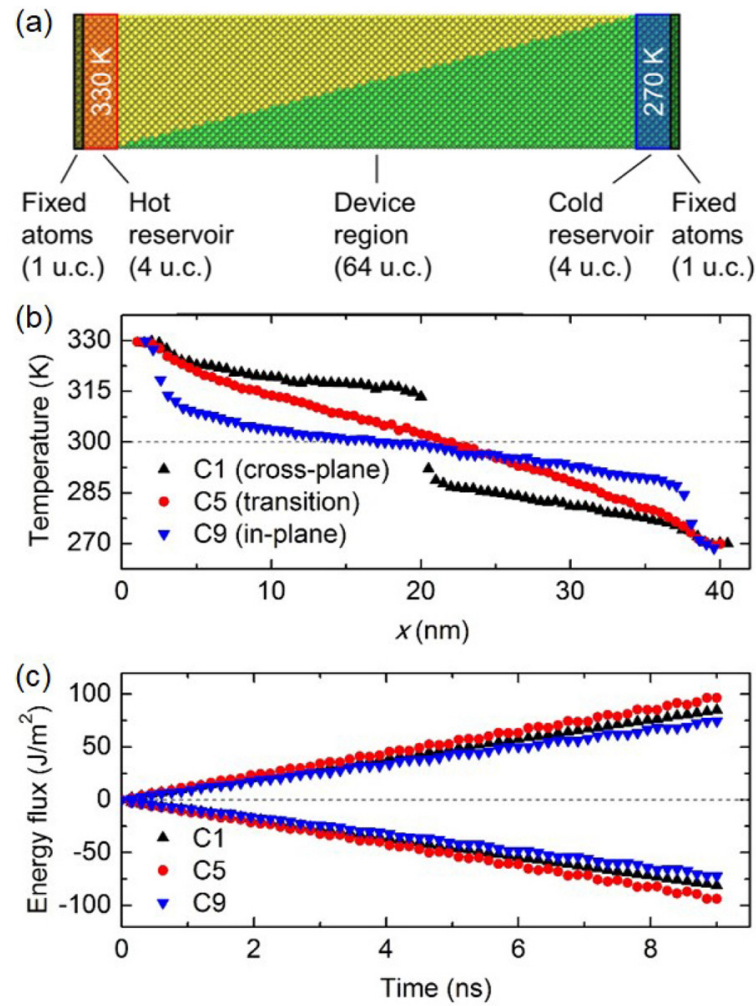

Fig. 2. (a) Illustration of the molecular dynamics simulation setup using C5 at $300 \mathrm{~K}$ as an example. The simulation domain contains fixed atoms, hot/cold reservoirs, and a device region. The length of each region along the $x$ direction (see Fig. 1(C1)) is given in the brackets as the number of unit cells (u.c.). (b) Typical temperature profiles for C1, C5, and C9 at $300 \mathrm{~K}$. (c) The energy flux profiles corresponding to (b).

Whereas the temperature profiles of $\mathrm{C} 1$ and C9 exhibit a large temperature jump at the $\mathrm{Si} / \mathrm{Ge}$ interface and at regions close to the thermal reservoirs, respectively, the temperature profile of $\mathrm{C} 5$ is smooth over the entire simulation domain. This suggests that C5 could be a better scheme for thermal transport. Fig. 2 (c) presents the thermal energy fluxes at the hot and cold reservoirs corresponding to the temperature profiles in Fig. 2(b). The linear, symmetric energy flux profiles confirm the stability of the simulations. It is clear that under the same temperature bias (i.e., $\Delta T=60 \mathrm{~K}$ ) the heat transfer rate decreases in the order: $\mathrm{C} 5>\mathrm{C} 1>\mathrm{C} 9$. This again suggests that $\mathrm{C} 5$ is a better scheme for thermal transport than $\mathrm{C} 1$ and $\mathrm{C}$.

To better assess the thermal transport properties of the different configurations, we calculate their thermal conductances as

$G^{\prime \prime}=\frac{1}{A_{\mathrm{c}} \Delta T}\left|\frac{\mathrm{dQ}}{\mathrm{dt}}\right|$,

where $A_{\mathrm{c}}$ is the cross-sectional (normal to the $x$-axis) area of the nanostructure, and $\Delta T$ is the temperature difference between the hot and cold reservoirs (i.e., $60 \mathrm{~K}$ ), $Q$ is the amount of energy added to the hot (or extracted from the cold) thermal reservoir, and $t$ is time. In fact, $|\mathrm{d} Q / \mathrm{d} t| / A_{\mathrm{c}}$ represents the magnitude of the heat flux (i.e., the slope of the data in Fig. 2(c)). Because $\mathrm{Si}$ and Ge have different, temperaturedependent coefficients of thermal expansion [27,29], $A_{\mathrm{c}}$ varies slightly with $x$. In this work, we adopt the $A_{\mathrm{c}}$ at the "A-A" cross section (Fig. 1(C1)).

Fig. 3(a) shows the thermal conductances of C1-C9 at 300 and $600 \mathrm{~K}$. At $300 \mathrm{~K}$, the thermal conductances of $\mathrm{C} 1$ and $\mathrm{C} 9$ are $(1.57 \pm 0.01) \times 10^{8}$ and $(1.60 \pm 0.02) \times 10^{8} \mathrm{~W} / \mathrm{m}^{2}-\mathrm{K}$, respectively. The slightly smaller thermal conductance of $\mathrm{C} 1$ than that of $\mathrm{C} 9$ results from the slightly bigger $A_{\mathrm{c}}$ of $\mathrm{C} 1$. The intermediate configurations exhibit higher thermal conductances than the cross-plane and in-plane 

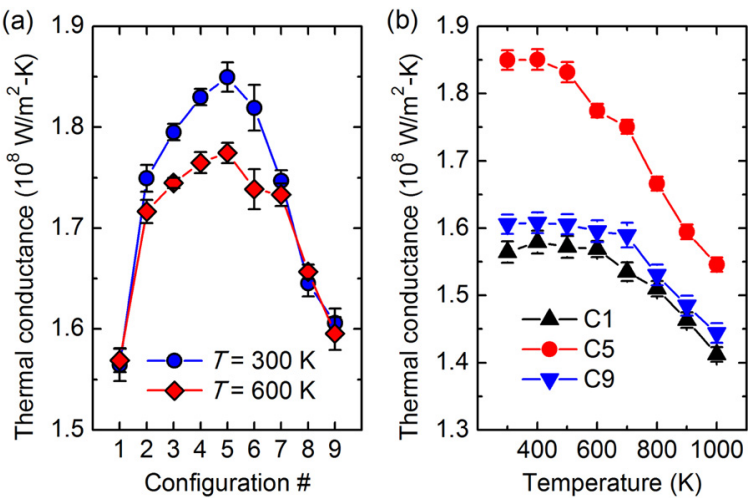

Fig. 3. (a) Thermal conductances of the nine $\mathrm{Si} / \mathrm{Ge}$ nanostructures (see Fig. 1(C1)-(C9)) at 300 and $600 \mathrm{~K}$. (b) Variation of the thermal conductances of $\mathrm{C} 1, \mathrm{C} 5$, and C9 with temperature.

configurations, achieving a maximum of $(1.85 \pm 0.02) \times 10^{8} \mathrm{~W} / \mathrm{m}^{2}-\mathrm{K}$ at $\mathrm{C} 5$. These results confirm that C5 is indeed the best scheme among the nine for thermal transport. Similar results are observed at $600 \mathrm{~K}$, although the difference between the thermal conductances of $\mathrm{C} 1$ and $\mathrm{C} 5$ reduces to $13 \%$.

We further examine the temperature dependence of the thermal conductances of $\mathrm{C} 1, \mathrm{C} 5$, and $\mathrm{C} 9$, which are three characteristic configurations. Fig. 3(b) shows their thermal conductances in the temperature range from 300 to $1000 \mathrm{~K}$. The thermal conductances of $\mathrm{C} 1$ and C9 have a similar temperature dependence: they remain robust up to about $600 \mathrm{~K}$ and then decrease with increasing temperature at a similar rate. In contrast, the thermal conductance of C5 has a plateau only up to about $400 \mathrm{~K}$ and then decreases with increasing temperature at a larger rate. In the entire temperature range, the order of the thermal conductances (i.e., C5 > C9 > C1) remains unchanged.

To understand the thermal conductance results, we evaluate the local VDOS at seven regions (Fig. 4(a)), which are 4 u.c.-thick slabs at $x=1,5,20,35,50,65$, and 69 u.c., respectively. The VDOS is calculated as [30]

$\operatorname{VDOS}(f)=\int_{-\infty}^{\infty} \frac{<\sum_{i=0}^{N} \mathbf{v}_{i}(t) \cdot \mathbf{v}_{i}(0)>}{<\sum_{i=0}^{N} \mathbf{v}_{i}(0) \cdot \mathbf{v}_{i}(0)>} e^{-i 2 \pi \mathrm{ft}} \mathrm{dt}$,

where $f$ is the vibration frequency, $N$ is the number of atoms in the considered region, " $\langle\cdots\rangle$ " is autocorrelation, $\mathbf{v}_{i}$ is the velocity vector of atom $i$, and $t$ is time. We calculate the VDOS by using the velocity data from the last $0.05 \mathrm{~ns}$ of each simulation. Because the phonon population increases with increasing temperature and the different regions have moderately different temperatures, we report the intrinsic VDOS rather than the normalized one.

As an example, the VDOS profiles for C5 at $300 \mathrm{~K}$ at the seven regions are shown in Fig. 4(b). From left to right (i.e., from Region 0 to Region 6), the most prominent peak in the VDOS profile gradually switches from around 15 to $9 \mathrm{THz}$, which are the characteristic peaks of Si and Ge, respectively [25].

For thermal transport across an interface, it has been shown that the oVDOS on both sides of the interface plays an important role [13,21]. To examine whether this explanation applies to the configurations considered in this work, we calculate the oVDOS values in Regions 0 and 1 (labeled "0-1"), 1-5 (labeled "1-5"), 5 and 6 (labeled " $5-6$ "), and 0-6 (labeled "0-6"). For example, the oVDOS in Regions 0 and 1 is computed as

$\operatorname{oVDOS}_{0-1}=\int_{0}^{f_{\max }} \min \left[\operatorname{VDOS}_{0}(f), \operatorname{VDOS}_{1}(f)\right] \mathrm{d} f$,

where $f_{\max }$ is the cutoff frequency in the VDOS spectrum. In this work, we choose $f_{\max }=20 \mathrm{THz}$. Physically, oVDOS $0_{-1}$ represents the number of phonons that coexist in Regions 0 and 1 (subject to a correction
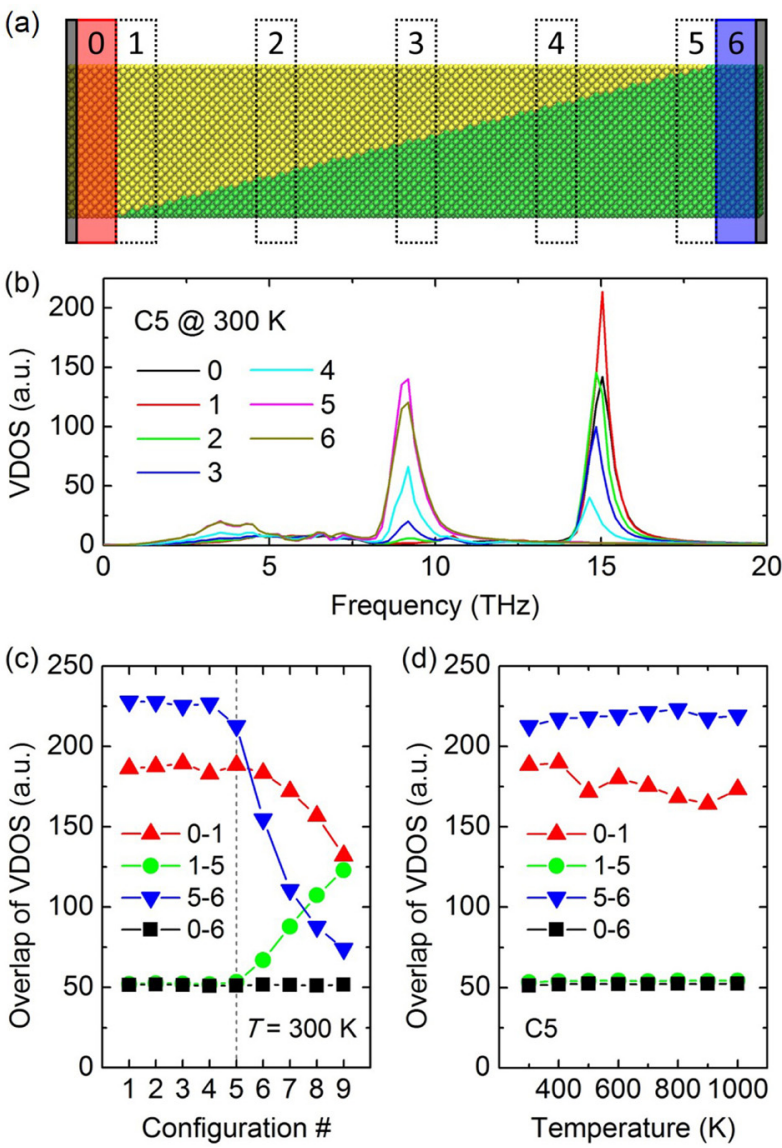

Fig. 4. (a) Illustration of the seven regions used for calculating the vibrational density of states (VDOS). (b) VDOS profiles in seven regions for C5 at $300 \mathrm{~K}$. (c) Overlap of VDOS for C1-C9 at $300 \mathrm{~K}$. " $m-n$ " denotes the overlap of VDOS profiles from Region- $m$ to Region- $n$. (d) Overlap of VDOS for C5 as a function of temperature.

factor related to the Bose-Einstein distribution).

Fig. 4(c) shows the four overlaps in C1-C9 at $300 \mathrm{~K}$. From C1 to C5, all the four overlaps remain nearly constant. From C5 to C9, oVDOS ${ }_{0-1}$ and oVDOS ${ }_{5-6}$ decrease dramatically, especially the latter; conversely,

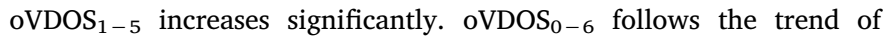
oVDOS $_{1-5}$ from C1 to C5 and is slightly smaller than the latter, implying the dominating role of oVDOS $_{1-5}$ over oVDOS $_{0-1}$ and oVDOS $_{5-6}$. From C5 to C9, oVDOS ${ }_{0-6}$ deviates from the trend of oVDOS $_{1-5}$ and remains nearly constant. Although the thermal conductance data from C5 to C9 (Fig. 3(a)) could be explained by the dominating effects of oVDOS $_{0-1}$ and oVDOS $_{5-6}$ over that of oVDOS $_{1-5}$, the data from C1 to C5 cannot be explained by the oVDOS results in Fig. 4(c). Furthermore, the oVDOS results for C5 in the temperature range from 300 to $1000 \mathrm{~K}$ (Fig. 4(d)) exhibit no correlation with the thermal conductance data in Fig. 3(b). Therefore, considering the oVDOS does not provide a satisfactory explanation to the thermal conductance data in Fig. 3.

We move on to consider the integrated VDOS (intVDOS) in the seven regions (Fig. 4(a)). For example, the intVDOS in Region 0 is calculated as

$\operatorname{intVDOS}{ }_{0}=\int_{0}^{f_{\max }} \operatorname{VDOS}_{0}(f) \mathrm{d} f$.

Physically, intVDOS ${ }_{0}$ represents the number of phonons that exist in Regions 0 (subject to a correction factor related to the Bose-Einstein distribution).

Fig. 5(a) shows the seven intVDOS values in C1-C9 at $300 \mathrm{~K}$, whereas Fig. 5(b) shows the seven intVDOS values for C5 in the 

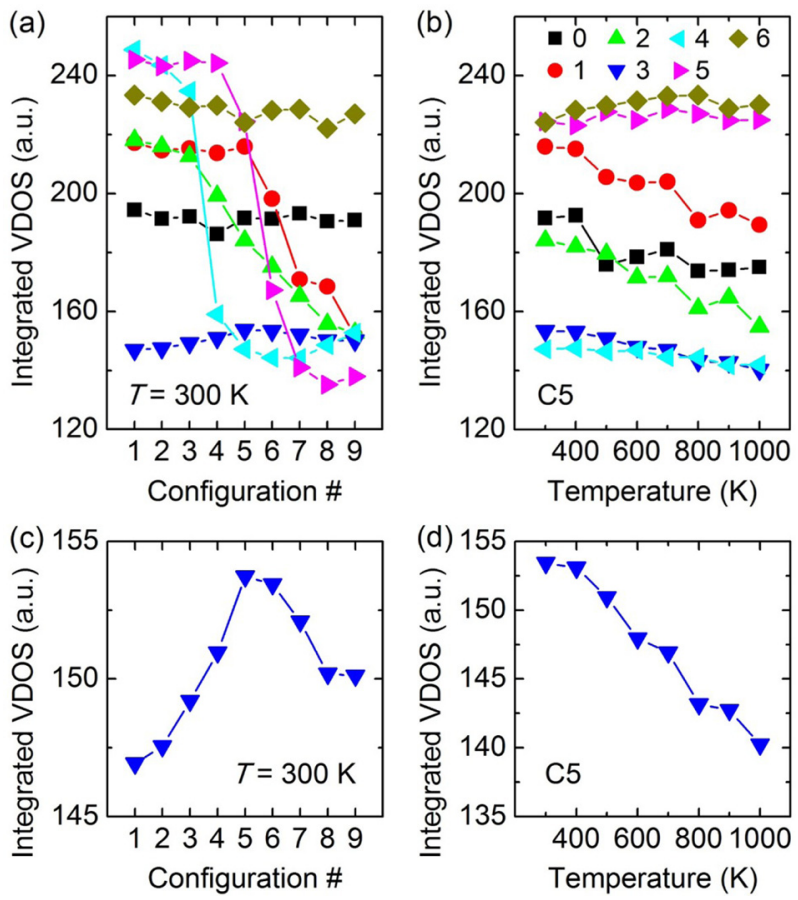

Fig. 5. (a) Integrated VDOS in seven regions for C1-C9 at $300 \mathrm{~K}$ (see (b) for the figure legend). (b) Integrated VDOS in seven regions for C5 as a function of temperature. (c) Integrated VDOS in Region 3 for C1-C9 at $300 \mathrm{~K}$. (d) Integrated VDOS in Region 3 for C5 as a function of temperature.

temperature range from 300 to $1000 \mathrm{~K}$. In both subfigures the intVDOS profiles in the seven regions exhibit distinct trends. Surprisingly, the profiles of the intVDOS 3 data in Fig. 5(a) and (b) closely resemble those of the thermal conductance data in Fig. 3(a) and (b), respectively, which are more clearly seen in Fig. 5(c) and (d). This suggests that intVDOS ${ }_{3}$ could be used as a qualitative criterion for characterizing the thermal conductance of the nanostructures considered in this work. The special role of intVDOS 3 is not unexpected because Region 3 lies in the middle of the simulation domain so that the influences of the thermal reservoirs on the VDOS profile are minimized or cancelled out in this region. In fact, the intermediate temperature in Region 3 (Fig. 2(b)) is an evident confirmation of the minimization or cancellation effect.

Because the $\mathrm{Si} / \mathrm{Ge}$ interfaces have different orientations in $\mathrm{C} 1-\mathrm{C} 9$, it is worth analyzing the cross-sectional heat flux distributions in C1-C9 to understand how the interface orientation affects the heat flow. The heat flux vector $\vec{J}$ is computed as $[12,31]$

$\vec{J}=\frac{1}{V}\left\{\sum_{i} \vec{v}_{i} e_{i}+\frac{1}{2} \sum_{i, j, i \neq j} \vec{r}_{\mathrm{ij}}\left(\vec{F}_{\mathrm{ij}} \cdot \vec{v}_{j}\right)+\sum_{i, j, k} \vec{r}_{\mathrm{ij}}\left[\vec{F}_{j}(\mathrm{ijk}) \cdot \vec{v}_{j}\right]\right\}$,

where $V$ is the total volume of the considered atoms, $\vec{v}_{i}$ and $e_{i}$ are the velocity and energy (potential and kinetic) of atom $i, \vec{r}_{\mathrm{ij}}$ is the distance between atoms $i$ and $j$, and $\vec{F}_{\mathrm{ij}}$ and $\vec{F}_{\mathrm{ijk}}$ are the two/ three-body forces between/among different atoms $(i, j$, or $k)$. To minimize the effect of the thermal reservoirs, we consider the atoms in the middle 44 u.c. region (i.e., at least 10 u.c. away from the thermal reservoirs) in the summations in Eq. (5). Because periodic boundary conditions are applied in the $z$ direction, it suffices to consider the heat flux distribution in the $y$ direction. We obtain the distribution by dividing the cross section into 16 uniform subregions along the $y$ direction (see the schematic for $\mathrm{C} 1$ in Fig. 6) and calculating the $\vec{J}$ in each of them. A constant heat rate of $5.0 \mathrm{eV} / \mathrm{ps}$ is simultaneously added to the hot reservoir and extracted from the cold reservoir by directly rescaling the velocities of the atoms in the thermal reservoirs, which allows establishing a temperature difference around $60 \mathrm{~K}$ across the device region under steady state. Similar to previous studies $[12,31]$, we focus on the cross-sectional
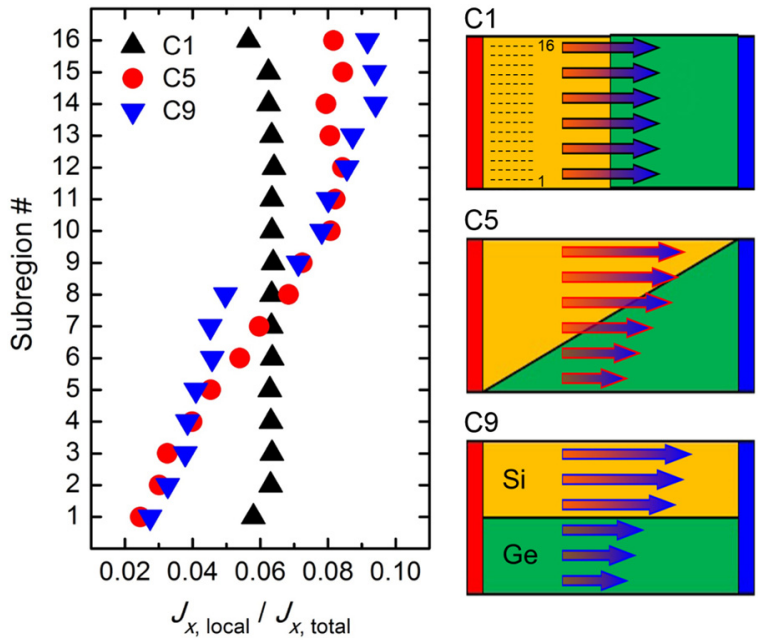

Fig. 6. Distribution of the local heat flux in $\mathrm{C} 1, \mathrm{C} 5$, and $\mathrm{C} 9$ along the $y$ direction. The schematics on the right illustrate the corresponding distributions. The 16 subregions are shown in the schematic for C1 by dashed lines.

distribution of the heat flux component in the main transport direction, i.e., $J_{x, \text { local }} / J_{x, \text { total }}$.

Fig. 6 shows the heat flux distributions in C1, C5, and C9 along with their schematics. Whereas the heat flux in $\mathrm{C} 1$ is distributed uniformly except for the slightly lower heat fluxes near the bottom and top surfaces, which are attributable to phonon localization [12,31], the distributions in C5 and C9 are apparently non-uniform, which can be explained by the tilted $\mathrm{Si} / \mathrm{Ge}$ interface and/or the higher thermal conductivity of $\mathrm{Si}$ than Ge. Our results clearly demonstrate that the interface orientation has a significant effect on the cross-sectional heat flux distribution in heterogeneous structures. For applications having a stringent requirement on the heat flux uniformity, $\mathrm{C} 1$ is the best among the nine configurations.

Lastly, we discuss the effects of the device and thermostat lengths on the thermal conductances of C1-C9. The bell-shaped trend seen in Fig. 3(a) results from an interplay among the thermal resistances of the reservoir/device interfaces, the $\mathrm{Si} / \mathrm{Ge}$ interface, and the $\mathrm{Si}$ and $\mathrm{Ge}$ materials. In this work, we focus on a fixed device length $\left(L_{\mathrm{D}}\right)$ of around $35.5 \mathrm{~nm}$, which is much smaller than the characteristic phonon mean free paths in crystalline $\mathrm{Si}$ and $\mathrm{Ge}$ (i.e., around 300 and $100 \mathrm{~nm}$, respectively $[32,33])$. As a result, the total thermal resistances are dominated by the resistances of the reservoir/device and $\mathrm{Si} / \mathrm{Ge}$ interfaces. As long as this condition is satisfied (e.g., for $L_{\mathrm{D}}<\sim 100 \mathrm{~nm}$ ), the thermal conductances in this work are expected to remain valid (at least qualitatively). In the limit of $L_{\mathrm{D}} \gg 300 \mathrm{~nm}$, where the thermal conductivities of $\mathrm{Si}$ and Ge have their bulk values and the thermal resistances of the reservoir/device and $\mathrm{Si} / \mathrm{Ge}$ interfaces become negligible compared to those of the $\mathrm{Si}$ and Ge materials, the thermal conductances of C1-C9 are expected to increase monotonically from ${G^{\prime}}^{\prime}{ }_{\mathrm{C} 1}=2 k_{\mathrm{Si}} k_{\mathrm{Ge}} /\left[L_{\mathrm{D}}\left(k_{\mathrm{Si}}+k_{\mathrm{Ge}}\right)\right]$ to ${G^{\prime}}_{\mathrm{C} 9}^{\prime}=\left(k_{\mathrm{Si}}+k_{\mathrm{Ge}}\right) /\left(2 L_{\mathrm{D}}\right)$, which are the effective thermal conductances of the serial and parallel thermal resistance networks, respectively. Substituting with $k_{\mathrm{Si}}=156 \mathrm{~W} / \mathrm{m}-\mathrm{K}$ and $k_{\mathrm{Ge}}=60 \mathrm{~W} / \mathrm{m}-\mathrm{K}$ [34], we obtain $G^{\prime{ }^{\prime} 1}=86.7 / L_{\mathrm{D}}$ and $G^{\prime{ }^{\prime} 9}=108 /$ $L_{\mathrm{D}}$. Therefore, $G^{\prime \prime}{ }_{\mathrm{C} 9}$ could be $25 \%$ higher than ${G^{\prime}}_{\mathrm{C} 1}$ in the long $L_{\mathrm{D}}$ limit. Additional studies are needed to investigate how the thermal conductances of C1-C9 change gradually from a bell-shaped trend to a monotonically increasing one as $L_{\mathrm{D}}$ increases. On the other hand, the length of the thermal reservoirs has an insignificant effect on the calculated thermal transport properties, provided that the target temperatures can be maintained within a small range by choosing appropriate thermostat parameters [35].

In conclusion, we have conducted NEMD simulations to investigate the thermal transport in nine $\mathrm{Si} / \mathrm{Ge}$ nanostructures with the interface 
orientation varying gradually from vertical to horizontal. The intermediate configurations show up to $18 \%$ higher thermal conductances than those of the cross-plane and in-plane configurations in the temperature range from 300 to $1000 \mathrm{~K}$. For all nanostructures, the thermal conductance decreases with increasing temperature, but the intermediate configurations show a larger rate of decrease. The thermal conductances are inconsistent with the oVDOS data but correlate well with the intVDOS in the middle region of the nanostructures. This work demonstrates that under the constraints of the same amount of materials and the same number of interfaces the intermediate configurations could be used to enhance the thermal transport across $\mathrm{Si} / \mathrm{Ge}$ nanostructures. It also shows that controlling the interface orientation is an effective way to regulate the heat flow in heterogeneous structures. The nanostructures considered in this work could be experimentally prepared by mating $\mathrm{Si}$ and $\mathrm{Ge}$ components grown or cut along specific crystalline planes.

\section{Data availability}

The raw/processed data required to reproduce these findings cannot be shared at this time due to technical or time limitations.

\section{Author contribution}

Zuyuan Wang: Conceptualization, Methodology, Software, Validation, Formal analysis, Investigation, Resources, Data curation, Writing - Original Draft, Writing - Reviewing \& Editing, Visualization, Project administration.

\section{Conflicts of interest}

The author declares no conflicts of interest.

\section{Acknowledgements}

The help of Bin Song in setting up LAMMPS is gratefully acknowledged. This work was supported by ERC AdG SmartPhon (Grant No.694977).

\section{References}

[1] D.J. Paul, Silicon-germanium strained layer materials in microelectronics, Adv. Mater. 11 (1999) 191-204.

[2] Y. Shiraki, N. Usami, Silicon-Germanium (SiGe) Nanostructures: Production, Properties and Applications in Electronics, Elsevier, 2011.

[3] R. Soref, Mid-infrared photonics in silicon and germanium, Nat. Photonics 4 (2010) 495.

[4] A. Malik, M. Muneeb, S. Pathak, Y. Shimura, J. Van Campenhout, R. Loo, G. Roelkens, Germanium-on-silicon midinfrared arrayed waveguide grating multiplexers, IEEE Photonics Technol. Lett. 25 (2013) 1805-1808.

[5] F. Aflatouni, B. Abiri, A. Rekhi, A. Hajimiri, Nanophotonic coherent imager, Opt. Express 23 (2015) 5117-5125.

[6] J.-s. Maa, J. Tang, J.-J. Lee, D. J. Tweet, and S. T. Hsu, Silicon/germanium superlattice thermal sensor, (2008), US Patent 7, 442, 599.

[7] L. Colace, G. Assanto, Germanium on silicon for nearinfrared light sensing, IEEE Photonics J. 1 (2009) 69-79.

[8] S. Volz, J. Saulnier, G. Chen, P. Beauchamp, Computation of thermal conductivity of si/ge superlattices by molecular dynamics techniques, Microelectron. J. 31 (2000) 815-819.

[9] R. Yang, G. Chen, M.S. Dresselhaus, Thermal conductivity modeling of core-shell and tubular nanowires, Nano Lett. 5 (2005) 1111-1115.

[10] H. Zhao, J.B. Freund, Phonon scattering at a rough interface between two fcc lattices, J. Appl. Phys. 105 (2009) 013515.

[11] L. Sun, J.Y. Murthy, Molecular dynamics simulation of phonon scattering at silicon/ germanium interfaces, J. Heat Transf. 132 (2010) 102403.

[12] M. Hu, K.P. Giapis, J.V. Goicochea, X. Zhang, D. Poulikakos, Significant reduction of thermal conductivity in si/ge core-shell nanowires, Nano Lett. 11 (2010) 618-623.

[13] Z. Tian, K. Esfarjani, G. Chen, Enhancing phonon transmission across a si/ge interface by atomic roughness: first-principles study with the green's function method, Phys. Rev. B 86 (2012) 235304.

[14] Y. Chalopin, K. Esfarjani, A. Henry, S. Volz, G. Chen, Thermal interface conductance in si/ge superlattices by equilibrium molecular dynamics, Phys. Rev. B 85 (2012) 195302.

[15] R.R. Kakodkar, J.P. Feser, A framework for solving atomistic phonon-structure scattering problems in the frequency do-main using perfectly matched layer boundaries, J. Appl. Phys. 118 (2015) 094301.

[16] K. Gordiz, A. Henry, Phonon transport at crystalline si/ge interfaces: the role of interfacial modes of vibration, Sci. Rep. 6 (2016) 23139.

[17] R. Venkatasubramanian, E. Siivola, T. Colpitts, In-plane thermoelectric properties of freestanding si/ge superlattice structures, Seventeenth International Conference on Thermoelectrics. Proceedings ICT98 (Cat. No. 98TH8365), IEEE, 1998, pp. 191-197.

[18] W. Liu, T. Borca-Tasciuc, J. Liu, K. Taka, K. Wang, M. Dresselhaus, G. Chen, Inplane thermoelectric properties of si/ge superlattice, Proceedings ICT2001. 20th International Conference on Thermoelectrics (Cat. No. 01TH8589), IEEE, 2001, pp. $340-343$.

[19] P.E. Hopkins, Thermal transport across solid interfaces with nanoscale imperfections: effects of roughness, disorder, dislocations, and bonding on thermal boundary conductance, ISRN Mech. Eng. 2013 (2013).

[20] R.R. Kakodkar, J.P. Feser, Probing the validity of the diffuse mismatch model for phonons using atomistic simulations, Phys. Rev. B 95 (2017) 125434.

[21] X. Zhang, J. Jiang, Thermal conductivity of zeolitic imidazolate framework-8: a molecular simulation study, J. Phys. Chem. C 117 (2013) 18441-18447.

[22] S. Plimpton, Fast parallel algorithms for short-range molecular dynamics, J. Comput. Phys. 117 (1995) 1-19.

[23] Z. Wang, T. Feng, X. Ruan, Thermal conductivity and spectral phonon properties of freestanding and supported silicene, J. Appl. Phys. 117 (2015) 084317.

[24] J. Tersoff, Modeling solid-state chemistry: interatomic potentials for multicomponent systems, Phys. Rev. B 39 (1989) 5566.

[25] S. Wei, M. Chou, Phonon dispersions of silicon and germanium from first-principles calculations, Phys. Rev. B 50 (1994) 2221.

[26] H.J. Berendsen, J.v. Postma, W.F. van Gunsteren, A. DiNola, J.R. Haak, Molecular dynamics with coupling to an external bath, J. Chem. Phys. 81 (1984) 3684-3690.

[27] M. Straumanis, E. Aka, Lattice parameters, coefficients of thermal expansion, and atomic weights of purest silicon and germanium, J. Appl. Phys. 23 (1952) 330-334.

[28] Z. Wang, S. Safarkhani, G. Lin, X. Ruan, Uncertainty quantification of thermal conductivities from equilibrium molecular dynamics simulations, Int. J. Heat Mass Transf. 112 (2017) 267-278.

[29] D. Gibbons, Thermal expansion of some crystals with the diamond structure, Phys. Rev. 112 (1958) 136.

[30] J. Dickey, A. Paskin, Computer simulation of the lattice dynamics of solids, Phys. Rev. 188 (1969) 1407.

[31] Y. Wang, B. Qiu, X. Ruan, Edge effect on thermal transport in graphene nanoribbons: a phonon localization mechanism beyond edge roughness scattering, Appl. Phys. Lett. 101 (2012) 013101.

[32] Y. Ju, K. Goodson, Phonon scattering in silicon films with thickness of order 100 nm, Appl. Phys. Lett. 74 (1999) 3005-3007.

[33] J. Alvarez-Quintana, J. Rodriguez-Viejo, F. Alvarez, D. Jou, Thermal conductivity of thin single-crystalline germanium-oninsulator structures, Int. J. Heat Mass Transf. 54 (2011) 1959-1962.

[34] C. Glassbrenner, G.A. Slack, Thermal conductivity of silicon and germanium from 3 K to the melting point, Phys. Rev. 134 (1964) A1058.

[35] Z. Wang, Atomistic Simulations of Spectral Phonon Properties of Solids, Purdue University, 2017 Ph.D. Thesis. 